\title{
latrogenic atrial septal defect: reassurance or inquisitiveness
}

\author{
Niyada Naksuk ${ }^{1}$ - Samuel J. Asirvatham ${ }^{1,2}$ \\ Received: 29 March 2018 / Accepted: 3 April 2018 / Published online: 22 April 2018 \\ (C) Springer Science+Business Media, LLC, part of Springer Nature 2018
}

\section{Editorial comments}

Since pulmonary vein isolation for treatment of atrial fibrillation (AF) was introduced in 1990s, several unexpected complications, including atrioesophageal fistula, pulmonary vein stenosis, and stiff left atrial syndrome, have been discovered in those who apparently tolerated the procedure well. Iatrogenic atrial septal defect (iASD) following transseptal puncture is increasingly recognized and may be another nuance surrounding AF ablation unknown. In this issue of the Journal of Interventional Cardiovascular Electrophysiology, Linhart et al. [1] confirmed that iASD is more frequent than our previous estimates. In 102 patients who had undergone cryoballoon ablation with single transseptal puncture, transesophageal echocardiography performed at approximately 3year post-procedure revealed $37 \%$ persistent iASD. However, the authors highlighted that an absence of clinical complications or deterioration of echocardiographic parameters was an optimistic message to our community. Is this enough to reassure electrophysiologists and patients that persistent iASD, an unavoidable consequence after transseptal puncture, is a benign defect?

\section{Congenital defect}

Congenital atrial septal defect (ASD) and patent foramen ovale (PFO) are related to hemodynamic consequence, atrial arrhythmias, and systemic emboli in late adulthood. Guidelines recommend clinical and echo surveillance in patients with ASD, and closure is indicated for right atrial and right ventricular enlargement [2].

Samuel J. Asirvatham

asirvatham.samuel@mayo.edu

1 Division of Cardiovascular Diseases, Department of Medicine, Mayo Clinic, Rochester, MN, USA

2 Department of Pediatrics and Adolescent Medicine, Mayo Clinic, 200 First Street SW, Rochester, MN 55905, USA
A search for PFO is crucial in patients with cryptogenic stroke because closure of the PFO effectively reduces recurrent paradoxical emboli [3-5]. While PFO is so common in general population with a prevalence around $20-25 \%$, not all PFOs are associated with stroke. High-risk characteristics of PFO include moderate-to-large right-to-left shunt, large PFO size (more than $8.0 \times 8.0 \mathrm{~mm}$ ) and concomitant atrial septal aneurysm [3-5]. Observational studies also found an association between PFO and paradoxical emboli in the setting of pre-existing cardiac implantable electronic devices (CIEDs) [6]. During transvenous lead extraction, debris surrounding the extracted leads can be dislodged through the preexisting PFO. The risk is likely augmented by general anesthesia or during the perioperative period which predisposes to right atrial pressure elevation [7].

Other clinical manifestations, including decompression sickness, may be related to PFO [8]. Expert consensus advises against continued diving in individuals with an intracardiac right-to-left shunt [9]. The association between migraine with aura and right-to-left shunt is controversial. Results from the only randomized controlled trial available did not support the benefit of PFO closure [10].

\section{3 latrogenic defect}

Transseptal puncture is widely performed during a percutaneous intervention to gain access to the left atrium and the left ventricle. Many studies confirmed that transseptal puncture leaves an unavoidable defect on the fossa ovalis. However, the reported incidence of iASD varies based on follow-up time and procedural characteristics. In patients who have undergone cryoballoon ablation delivered with 15 French sheath, the incidence of iASD is approximately $17-38 \%$ at $4-6$ months $[11,12]$ and $20-37 \%$ at $12-36$ months $[1,13-15]$. In comparison to radiofrequency ablation (RFA) technique with 8-8.5 French sheaths, 2\% [11] and 9-19\% iASD [15, 16] are observed at 4-6 months and 12-36 months, respectively. The prevalence of persistent iASD after Watchman left atrial appendage closure delivered with 14 French from a post 
hoc analysis of the PROTECT AF was $11 \%$ at 6 months and $7 \%$ at 12 months $[16,17]$.

\section{Shunting through the defect}

At present, leaving a defect after transseptal puncture is not considered a complication. Although shunting through iASD is documented universally, follow-up or closure is not routinely performed. The shunt is predominantly left-to-right in an otherwise structurally normal heart while right-to-left shunting can be observed in patients with underlying right atrial pressure elevation [12].

Hemodynamic consequence of iASD remains debatable. As suggested by the present study, the outcomes of iASD after AF ablation seem benign and presumably reassuring during 3year follow-up timeframe [1]. There are, however, collective reports of acute right ventricular failure requiring emergent iASD closure [18, 19].

The worst case scenario tends to develop significant hemodynamic consequence early post-transseptum. This is due to an acute change in cardiopulmonary flow physiology, particularly in patients with baseline left atrial pressure elevation and normal (or low) right atrial pressure as commonly seen in AF cases under general anesthesia. In this situation, an acutely created defect may lead to equalization of biatrial pressure, which could potentially result in no flow stage, and acute right ventricular failure in an unprepared ventricle [19].

Another unknown is long-term consequences in patients with persistent iASD who develop other comorbidities that predispose to either right or left filling pressure elevation. In view of AF ablation, one could expect to see more than one complication. Combination of pulmonary vein stenosis and iASD or stiff left atrial syndrome and iASD will likely enhance the risk of right ventricular failure.

\section{Emboli through the defect}

We now have learned from recent trials that a high-risk PFO is an etiology of previously labeled cryptogenic stroke and PFO closure significantly reduces risk of recurrent stroke [3-5]. Should the same line of management apply to persistent iASD? Most of the persistent iASD is relatively small, with an average size of $4.6-6.0 \mathrm{~mm}$, and thus without suggestive high-risk PFO characteristics. Further, majority of AF patients would need anticoagulation anyway and that seems to offset the risk of stroke [20]. However, this question is relevant in patients with $\mathrm{CHA}_{2} \mathrm{DS}_{2}-\mathrm{VAS}_{2}$ score of $0-1$ when anticoagulation is generally discontinued few months postablation. The PROTECT AF reported 7\% iASD after Watchman implant but none suffered from systemic emboli at 12-month follow-up [17]. In contrast to a cross-sectional study derived for a large national database, Madhavan et al. [20] found that patients with preexisting CIEDs who had undergone a transseptal procedure were at a higher risk of stroke with an overall risk of systemic embolism around 2 per 100 person-year. Noteworthy that the risk was mitigated by anticoagulation. Due to the nature of the study, the prevalence of iASD was not available to review, thus the causal relation cannot be derived with confidence.

Finally, silent cerebral embolism related to transseptal procedures and, in particular, pulmonary vein isolation, becomes increasingly recognized [21]. We are in need of well-designed studies to examine the interaction between persistent iASD, anticoagulation status, brain magnetic resonance imagingbased change, and cognitive function, which will allow us to better understand this complex and important issue.

\section{Good defect}

An observational study of patients with inoperable severe mitral regurgitation who had undergone MitralClip placement reported an immediate relief in left atrial pressure and volume among those with iASD [22]. The authors assumed that presence of iASD might be beneficial. This concept is in line with palliative atrial septostomy performed in patients with severe diastolic heart failure or stiff left atrial syndrome for the purpose of volume and pressure relief [23, 24]. Among AF patients who have these preexisting conditions, a persistent iASD may be a good defect, despite some risk of right ventricular failure [22].

\section{Dealing with the defect}

There may be few reasons against routine defect closure at time of the index procedure. Only a portion of patients has residual $\mathrm{iASD}$, and the defect tends to close spontaneously overtime [12]. Chan et al. observed the incidence of iASD after cryoballoon ablation significantly reduced from $38 \%$ at 6 months to $31 \%$ at 9 months [12]. Similarly after Watchman implant, iASD was found in $34 \%$ at 45 days and $7 \%$ at 1 year, respectively [17]. Of the remaining, only few cases may have physiological adverse effect. A substantial difference in left and right atrial pressure may lead to persistent iASD in some patients (for instance stiff left atrium) who could potentially benefit from maintaining the defect.

Additional procedure is not without risks. Recent randomized trials which enrolled patients with cryptogenic stroke observed the incidence of AF 4.6-6.6\% soon after PFO closure $[4,5]$. While AF was attributed to the procedure itself, whether iASD closure after AF ablation would lead to an increase in recurrent atrial arrhythmias is to be determined. 
Finally, closure of iASD may also lead to difficulty in redotransseptal punctured despite its feasibility.

\section{Screening the defect}

It is virtually impossible to offer routine surveillance for iASD because of lack of non-invasive method. Limited longitudinal studies make it difficult to recommend an optimal time when to screen since iASD tends to close overtime. In the contrary, the rate of catheter ablation for treatment of AF is rising significantly, even in the patient populations with more comorbidities [25]. Electrophysiologists therefore should expect to observe more of iASD and perhaps its related complications. The key is to exercise careful judgment on an individual basis when encountering worsening clinical symptoms in patients with a prior history of transseptal puncture and evaluating for the presence of iASD may be useful in these scenarios:

1. Patients who developed pulmonary hypertension (predispose to right-to-left shunting) presenting with worsening hypoxia

2. Patients with severely increased left atrial filling pressure (predispose to left-to-right shunting) with new onset right ventricular failure

3. Patients with $\mathrm{CHA}_{2} \mathrm{DS}_{2}-\mathrm{VAS}_{2}$ score of $0-1$ when discontinuation of anticoagulation is being considered

4. Patients with preexisting CIEDs who will encounter a circumstance that significantly elevates right atrial pressure (for instance undergoing general anesthesia), as well as, those who are undergoing a procedure involving endovascular lead manipulation

5. Patients who wish to scuba dive

Conclusively, the study by Linhart et al. [1] does not reassure our community, but raises inquisitiveness, as well as concern of unknowns surrounding the AF ablation. We shall closely observe this consequence and be ready to intervene when indicated.

\section{Preventing the defect}

It is noteworthy that persistent iASD seems more prevalent after cryoballoon ablation. Should cryoablation be discouraged? Higher risk of iASD may be due to a bigger and stiffer sheath for the cryoablation system (15 French) [26]. Although RFA requires two delivery sheaths for mapping and ablating (8-8.5 French), Hammerstingl et al. [16] noted less iASD with double transseptal puncture technique, compared to two catheters across a single transseptal site. Further, the reported incidence of iASD after MitralClip implant using a 22-24 French (the largest sheath for the septal crossing in all procedures) was less than that after cryoablation ablation (27\% at 1 year) [27]. Collectively, this data might suggest that manipulation across the septum is another predictor of persistent iASD. Placement of MitralClip or double transseptal puncture technique for RFA requires less active manipulation, while cryoablation sheath may cause more stretching and tearing of the septum especially when torqueing and turning are generally required to engage the right inferior pulmonary vein [26].

On the other hand, we must not ignore the benefits of cryoballoon ablation which offers simplification and overall shorter procedural time with similar efficacy to that of RFA [28]. Further, using cryoablation limits ability to create additional ablative lines in the left atrium, which, in turn, may reduce the risk of stiff left atrial syndrome predisposing to elevated pressure keeping persistent iASD in long-term.

An ultimate way to avoid persistent iASD is to give up the entire endocardial ablation and to consider alternative approaches. Autonomic system plays an important role in the pathogenesis of AF, although the effectiveness of autonomic modification for treatment of AF remains controversial [29]. Randomized trials tested the concept of standalone epicardial ablation in patients mostly with permanent $\mathrm{AF}$ who had recurrent $\mathrm{AF}$ after traditional endocardial approach proved reasonable efficacy [30, 31]. Catheter-free ablation is an active area of research with promising results [24-27]. Perhaps further advances in these novel approaches for treatment of AF may allow electrophysiologists to eliminate AF substrates and triggers without a need to enter the left atrium.

\section{References}

1. Markus Linhart JW, Florian Stöckigt, Annika T Kohlmann, Pia C Lodde, Lutz PT Linneborn, Thomas Beiert, Christoph Hammerstingl, Roger Borràs, Georg Nickenig, René P Andrié, Jan W Schrickel. High rate of persistent iatrogenic atrial septal defect after single transseptal puncture for cryoballoon pulmonary vein isolation. J Interv Card Electrophysiol. 2018.

2. Warnes CA, Williams RG, Bashore TM, Child JS, Connolly HM, Dearani JA, del Nido P, Fasules JW, Graham TP, Hijazi ZM, Hunt SA, King ME, Landzberg MJ, Miner PD, Radford MJ, Walsh EP, Webb GD. Acc/aha 2008 guidelines for the management of adults with congenital heart disease: Executive summary. <span class $="$ subtitle" $>A$ Report of the American College of Cardiology/American Heart Association Task Force on Practice Guidelines (Writing Committee to Develop Guidelines for the Management of Adults With Congenital Heart Disease): $<$ em $>$ Developed in Collaboration With the American Society of Echocardiography, Heart Rhythm Society, International Society for Adult Congenital Heart Disease, Society for Cardiovascular Angiography and Interventions, and Society of Thoracic Surgeons $</$ em $></$ span $>$. 2008;118:2395-2451.

3. Saver JL, Carroll JD, Thaler DE, Smalling RW, MacDonald LA, Marks DS, et al. Long-term outcomes of patent foramen ovale 
closure or medical therapy after stroke. N Engl J Med. 2017;377: 1022-32.

4. Søndergaard L, Kasner SE, Rhodes JF, Andersen G, Iversen HK, Nielsen-Kudsk JE, et al. Patent foramen ovale closure or antiplatelet therapy for cryptogenic stroke. N Engl J Med. 2017;377:1033-42.

5. Mas J-L, Derumeaux G, Guillon B, Massardier E, Hosseini H, Mechtouff L, et al. Patent foramen ovale closure or anticoagulation vs. Antiplatelets after stroke. N Engl J Med. 2017;377:1011-21.

6. DeSimone CV, Friedman PA, Noheria A, Patel NA, DeSimone DC, Bdeir $\mathrm{S}$, et al. Stroke or transient ischemic attack in patients with transvenous pacemaker or defibrillator and echocardiographically detected patent foramen ovale. Circulation. 2013;128:1433-41.

7. Dayal NB, Narata AP, Burri H. Acute stroke from paradoxical embolism of dense fibrous tissue following pacemaker lead extraction: salvation by mechanical thrombectomy. Clinical Case Reports. 2016;4:158-61.

8. Wilmshurst PT, Byrne JC, Webb-Peploe MM. Relation between interatrial shunts and decompression sickness in divers. Lancet (London, England). 1989;2:1302-6.

9. British thoracic society guidelines on respiratory aspects of fitness for diving. Thorax. 2003;58:3-13.

10. Dowson A, Mullen MJ, Peatfield R, Muir K, Khan AA, Wells C, et al. Migraine intervention with starflex technology (mist) trial: a prospective, multicenter, double-blind, sham-controlled trial to evaluate the effectiveness of patent foramen ovale closure with starflex septal repair implant to resolve refractory migraine headache. Circulation. 2008;117:1397-404.

11. Cronin EM, Collier P, Wazni OM, Griffin BP, Jaber WA, Saliba WI. Persistence of atrial septal defect after cryoballoon ablation of atrial fibrillation. J Am Coll Cardiol. 2013;62:1491-2.

12. Chan N-Y, Choy C-C, Lau C-L, Lo Y-K, Chu P-S, Yuen H-C, et al. Persistent iatrogenic atrial septal defect after pulmonary vein isolation by cryoballoon: an under-recognized complication. EP Europace. 2011;13:1406-10.

13. MUGNAI G, SIEIRA J, CICONTE G, HERVAS MS, IRFAN G, SAITOH Y, et al. One year incidence of atrial septal defect after pv isolation: a comparison between conventional radiofrequency and cryoballoon ablation. Pacing Clin Electrophysiol. 2015;38:104957.

14. Sieira J, Chierchia GB, Di Giovanni G, Conte G, De Asmundis C, Sarkozy A, et al. One year incidence of iatrogenic atrial septal defect after cryoballoon ablation for atrial fibrillation. J Cardiovasc Electrophysiol. 2014;25:11-5.

15. Mugnai G, Sieira J, Ciconte G, Hervas MS, Irfan G, Saitoh Y, et al. One year incidence of atrial septal defect after pv isolation: a comparison between conventional radiofrequency and cryoballoon ablation. Pacing Clin Electrophysiol: PACE. 2015;38:1049-57.

16. Hammerstingl C, Lickfett L, Jeong KM, Troatz C, Wedekind JA, Tiemann K, et al. Persistence of iatrogenic atrial septal defect after pulmonary vein isolation-an underestimated risk? Am Heart J. 2006;152(362): e361-5.

17. Singh SM, Douglas PS, Reddy VY. The incidence and long-term clinical outcome of iatrogenic atrial septal defects secondary to transseptal catheterization with a $12 \mathrm{f}$ transseptal sheath. Circ Arrhythm Electrophysiol. 2011;4:166-71.

18. Ussia GP, Cammalleri V, Marchei M, Sergi D, De Vico P, Muscoli $\mathrm{S}$, Sarkar K, Romeo F. Hemodynamic patterns of residual interatrial communication after transcatheter mitraclip repair. J Cardiovasc Med(Hagerstown, Md.). 2014;15:343-349.

19. Yousuf MA, Haq S, O’Donnell RE, Attari M. Hemodynamically significant atrial septal defect after atrial fibrillation ablation: A hole to remember. Heart Rhythm.12:1987-1989.

20. Madhavan M, Yao X, Sangaralingham LR, Asirvatham SJ, Friedman PA, McLeod CJ, et al. Ischemic stroke or systemic embolism after transseptal ablation of arrhythmias in patients with cardiac implantable electronic devices. J Am Heart Assoc. 2016;5:e003163.

21. Gaita F, Caponi D, Pianelli M, Scaglione M, Toso E, Cesarani F, et al. Radiofrequency catheter ablation of atrial fibrillation: a cause of silent thromboembolism? Magnetic resonance imaging assessment of cerebral thromboembolism in patients undergoing ablation of atrial fibrillation. Circulation. 2010;122:1667-73.

22. Smith T, McGinty P, Bommer W, Low RI, Lim S, Fail P, et al. Prevalence and echocardiographic features of iatrogenic atrial septal defect after catheter-based mitral valve repair with the mitraclip system. Catheter Cardiovasc Interv : Off J Soc Cardiac Angiography Interv. 2012;80:678-85.

23. Chandrashekar P, Park JY, Al-Hijji MA, Reddy YNV, Zack CJ, Reeder GS, et al. Atrial septostomy to treat stiff left atrium syndrome. Circ Heart Fail. 2017;10:e004160.

24. Kaye DM, Hasenfuss G, Neuzil P, Post MC, Doughty R, Trochu $\mathrm{JN}$, et al. One-year outcomes after transcatheter insertion of an interatrial shunt device for the management of heart failure with preserved ejection fraction. Circ. Heart Fail. 2016;9:e003662.

25. Kneeland PP, Fang MC. Trends in catheter ablation for atrial fibrillation in the United States. J Hosp Med : Off Publ Soc Hosp Med. 2009;4:E1-5.

26. Alkhouli M, Sarraf M, Zack CJ, Holmes DR, Rihal CS. Iatrogenic atrial septal defect following transseptal cardiac interventions. Int $\mathrm{J}$ Cardiol. 2016;209:142-8.

27. Smith T, McGinty P, Bommer W, Low RI, Lim S, Fail P, et al. Prevalence and echocardiographic features of iatrogenic atrial septal defect after catheter-based mitral valve repair with the mitraclip system. Catheter Cardiovasc Interv. 2012;80:678-85.

28. Kuck K-H, Brugada J, Fürnkranz A, Metzner A, Ouyang F, Chun $\mathrm{KRJ}$, et al. Cryoballoon or radiofrequency ablation for paroxysmal atrial fibrillation. N Engl J Med. 2016;374:2235-45.

29. Driessen AHG, Berger WR, Krul SPJ, van den Berg NWE, Neefs J, Piersma FR, et al. Ganglion plexus ablation in advanced atrial fibrillation: The afact study. J Am Coll Cardiol. 2016;68:1155-65.

30. Boersma LVA, Castella M, van Boven W, Berruezo A, Yilmaz A, Nadal M, Sandoval E, Calvo N, Brugada J, Kelder J, Wijffels M, Mont L. <span hwp:Id="article-title-1" class="article-title">atrial fibrillation catheter ablation versus surgical ablation treatment (fast) $</$ span $><$ span hwp:Id="article-title-29" class="sub-articletitle">clinical perspective $</$ span $>$. A 2-Center Randomized Clinical Trial. 2012;125:23-30.

31. POKUSHALOV E, ROMANOV A, ELESIN D, BOGACHEVPROKOPHIEV A, LOSIK D, BAIRAMOVA S, et al. Catheter versus surgical ablation of atrial fibrillation after a failed initial pulmonary vein isolation procedure: a randomized controlled trial. J Cardiovasc Electrophysiol. 2013;24:1338-43. 